\title{
A DEP-BASED LAB-ON-A-CHIP SYSTEM FOR THE DETECTION OF MULTIDRUG RESISTANCE IN K562 LEUKEMIA CELLS
}

\author{
Y. Demircan Yalçın ${ }^{1,3}$, G. Özkayar ${ }^{2,3}$, E. Özgür ${ }^{3}, U$. Gündüz ${ }^{5}$ and H. Külah ${ }^{1,3,4}$ \\ ${ }^{1}$ Department of Electrical and Electronics Engineering, METU, Ankara, Turkey \\ ${ }^{2}$ Graduate School of Natural and Applied Sciences/ Biomedical Engineering, METU, Ankara, Turkey \\ ${ }^{3}$ Mikro Biyosistemler Inc., Ankara, Turkey \\ ${ }^{4}$ METU-MEMS Research and Application Center, Ankara, Turkey \\ ${ }^{5}$ Department of Biology, METU, Ankara, Turkey
}

\begin{abstract}
This study presents a DEP-based lab-on-a-chip (LOC) system for label-free detection of multidrug resistant (MDR) K562 leukemia cells in a cell mixture, consisting of red blood cells (RBCs) and MDR-K562 cells, for the first time in the literature. The system consists of 2 consecutive DEP units, one for the depletion of RBCs and the other for capturing of MDR-K562 cells. RBCs are depleted by $60 \%$ in the first unit. In the second unit, MDR-K562 cell detection is performed with $100 \%$ selectivity at a flow rate of $10 \mu \mathrm{l} / \mathrm{min}$ and at $20 \mathrm{~V}_{\mathrm{pp}}$ in a cell mixture, containing less than $25 \%$ MDR-K562 cells.
\end{abstract}

\section{INTRODUCTION}

Multidrug resistance (MDR) is a condition enabling a cancer cell to resist distinct drugs of a wide variety of structure and function targeted at eradicating the cell. In case of MDR, patients do not respond chemotherapy so MDR detection in early stages of cancer is critical to envisage the most appropriate treatment and to speed up the recovery period [1].

Resistance to drugs is effectuated by some different mechanisms: (1) Drug efflux by membrane-associated proteins (Pglycoprotein (P-gp) and multidrug resistance-associated protein (MRP)), (2) drug elimination by exocytosis, (3) changing of drug targets, including DNA topoisomerase II, (4) enhancement of detoxification of compounds by glutathione system, and (5) overexpression of human major vault protein [2]. Although there are sensitive methods for MDR detection, they require expensive laboratory equipment and trained personnel. Moreover, they are time consuming due to labeling procedures and cannot be applied for frequent use during prognosis, because of the side effects [1, 3]. Hence, a cost effective, label-free, and user friendly method is needed to provide effective diagnosis of MDR in cancer cells, without compromising the sensitivity and specificity.

Dielectrophoresis (DEP) is a MEMS-enabled technique used to manipulate particles based on different sizes and/or dielectric properties under non-uniform electric field. Recent studies show that DEP is a potential tool to be applied in rapid MDR detection in a label-free manner $[1,4-7]$. It is reported that the cytoplasmic conductivity of MDR-K562 cells are 2.17 times higher than drug sensitive K562 cells, as analyzed by DEP collection spectra [4]. This variation is most probably associated with the overexpression of P-gp, modulating the volume-activated $\mathrm{Cl}^{-}$channels [8]. Coley et al. also reported that human breast cancer cells (MCF7) and its MDR progenies (MCF7-TaxR, MCF7-DoxR and MCF7-MDR1) have different cytoplasmic conductivities (MCF7TaxR $<$ MCF7 $<$ MCF7-MDR $1<$ MCF7-DoxR) [5]. We previously showed that imatinib resistant K562 cells could be trapped utilizing DEP, while the sensitive ones were not affected under the same experimental conditions [6]. We also reported that the same system is capable of detecting MDR in K562 cells at a flow rate of $10 \mu \mathrm{l} / \mathrm{min}$ by applying $5 \mathrm{~V}_{\mathrm{pp}}$, with $100 \%$ selectivity in a mixture containing less than $1 \%$ resistant cells [1]. In addition, we also revealed that DEP can be utilized for the detection of the level of resistance in K562 cells without requiring the examination of P-gp dynamic functions [7].

In this study, an LOC system has been developed for the detection of MDR in K562 leukemia cells, by integrating a DEPbased RBC depletion unit with an MDR-K562 cell detection unit. Results showed that MDR detection in K562 cells was achieved with $100 \%$ selectivity, even the resistant cells constituted less than $25 \%$ of overall population.

\section{THEORETICAL BACKGROUND DEP Theory}

DEP is an electrical particle manipulation technique defined as the relative motion of particles and medium under non-uniform electric field. The movement of particles is a result of the timeaveraged DEP force, which is defined for a homogenous spherical particle as [1]:

$$
\left\langle\mathrm{F}_{\mathrm{DEP}}\right\rangle=2 \pi \varepsilon_{\mathrm{m}} \mathrm{r}^{3} \operatorname{Re}\left(\mathrm{F}_{\mathrm{CM}}\right) \nabla \mathrm{E}_{\mathrm{rms}}^{2}
$$

where, $\varepsilon_{m}$ is the medium permittivity, $r$ is the particle radius, $\operatorname{Re}\left(f_{C M}\right)$ is the real part of Clausius-Mossotti factor of the particle, and $\nabla E_{r m s}^{2}$ is the gradient of external electric field.

$f_{C M}$ carries information about the particle and the medium dielectric properties, and the frequency dependence of DEP force. For spherical particles, $f_{C M}$ is formulized as [1]:

$$
f_{C M}=\frac{\varepsilon_{p}^{*}-\varepsilon_{m}^{*}}{\varepsilon_{p}^{*}+2 \varepsilon_{m}^{*}}
$$

where $\varepsilon_{p}^{*}$ and $\varepsilon_{m}^{*}$ are the complex permittivities of the particle and the suspending medium, respectively.

Complex permittivities of particle and medium are frequency dependent variables. Therefore, based on applied frequency, the relation between $\varepsilon_{p}^{*}$ and $\varepsilon_{m}^{*}$ changes. If $\varepsilon_{p}^{*}>\varepsilon_{m}^{*}$, particle is pulled towards stronger electric field region, observing positive DEP (pDEP) force. If $\varepsilon_{p}^{*}<\varepsilon_{m}^{*}$, particle is pushed towards weaker electric field region, observing negative DEP (nDEP) force. Utilizing Eq. 2, the frequency at which the particle observes no net DEP force (i.e., $\left.\operatorname{Re}\left(f_{C M}\right)=0\right)$ can be calculated. This frequency is named as crossover frequency $\left(f_{\text {cross }}\right)$.

Particles with similar sizes but different $f_{\text {cross }}$ values can be separated by $\mathrm{pDEP}$ or $\mathrm{nDEP}$ approach, at a certain signal frequency. If $f_{\text {cross }}$ values of particles are not different enough for separation, size-based separation of particles can be achieved through DEP, due to the dependence of DEP force to particle radius (Eq. 1).

\section{Cell Modeling}

$\operatorname{Re}\left(f_{C M}\right)$ depends on particle and medium dielectric properties. Medium properties can be attuned but particle dielectric properties are out of control and should be measured. After the 
measurement of dielectric properties of particles, they should be modeled to choose the proper structures for DEP operation.

Biological cell membrane and cytoplasm have different dielectric properties. These properties provide cell-specific characteristics as cells vary in their molecular content. This can be utilized as a discriminatory feature in DEP cell identification, manipulation or separation [1]. In this study, single shell cell modeling was utilized. Effective complex permittivity (Eq. 3) is obtained with this technique and it is used instead of particle complex permittivity $\varepsilon_{p}^{*}$ in the expression of $f_{C M}$ (Eq. 2) [1]. $R e\left(f_{C M}\right)$ vs frequency characteristics of RBCs, drug sensitive and MDR-K562 cells were obtained in MATLAB (Fig. 1).

$$
\varepsilon_{e f f, p}^{*}=\varepsilon_{s}^{*}\left\{\frac{[(r+d) / r]^{3}+2\left[\left(\varepsilon_{i}^{*}-\varepsilon_{s}^{*}\right) / \varepsilon_{i}^{*}+2 \varepsilon_{s}^{*}\right]}{[(r+d) / r]^{3}-\left[\left(\varepsilon_{i}^{*}-\varepsilon_{s}^{*}\right) / \varepsilon_{i}^{*}+2 \varepsilon_{s}^{*}\right]}\right\}
$$

where, $d$ is the thickness of the cell membrane, $\varepsilon_{i}^{*}$ and $\varepsilon_{S}^{*}$ are the cell interior and shell complex permittivities, respectively.

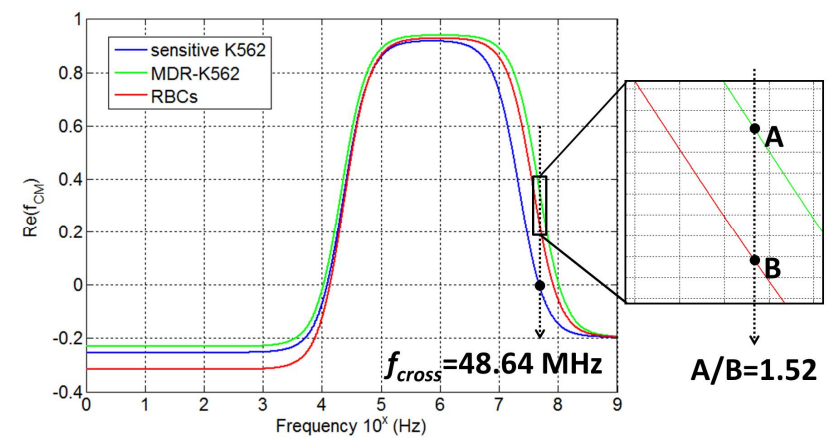

Figure 1: $\operatorname{Re}\left(f_{C M}\right)$ vs. frequency graphs of RBCs, drug sensitive and MDR-K562 cells in a medium with $2.5 \mathrm{mS} / \mathrm{m}$ conductivity and 78 permittivity coefficient.

According to this simulation, $f_{\text {cross }}$ of sensitive K562 cells was specified as $48.64 \mathrm{MHz}$ in a medium with the conductivity of 2.5 $\mathrm{mS} / \mathrm{m}$ as working frequency of MDR detection unit [1]. $\operatorname{Re}\left(f_{C M}\right)$ of MDR-K562 cells and RBCs were positive at that frequency and they should be pulled to higher electric field region through pDEP force, having different magnitudes. It should be distinguished that $\operatorname{Re}\left(f_{C M}\right)$ of MDR-K562 cells (point A) were 1.52 times higher than RBCs (point B) at $f_{\text {cross }}$ of sensitive K562 cells.

\section{DESIGN AND FABRICATION}

The device schematic was presented in Figure 2. RBC depletion unit is composed of a main channel $(1000 \mu \mathrm{m} \mathrm{W} \times 20$ $\mu \mathrm{m} \mathrm{H})$ and side channels $(500 \mu \mathrm{m} \mathrm{W} \times 20 \mu \mathrm{m} \mathrm{H})$ connected to each other via $8 \mu \mathrm{m}$ filtering gaps on the sidewalls to realize sizebased filtration.

Planar electrodes with $15^{\circ}$ angles were implemented throughout the channel to direct the cells through sidewall filtering gaps via DEP force. MDR detection unit has 2x29 3D-electrode arrays, with $40 \mu \mathrm{m}$ width and $15 \mu \mathrm{m}$ gaps in between, placed on the sidewalls of $300 \mu \mathrm{m} \times 20 \mu \mathrm{m}$ parylene microchannel. The channel also contains parylene posts for hydrodynamic focusing of cells to DEP traps. Isolation of both planar and 3D-electrodes from the solution is provided by means of thin parylene coating, reducing the Joule heating and cell damaging.

In RBC depletion unit, all cells are directed towards the sidewalls by sliding on the electrodes through pDEP force and hydrodynamic forces, at a frequency of $1 \mathrm{MHz}$. Small RBCs pass through the filters, while cancer cells continue through the main channel.

Based on the dielectric simulations, $f_{\text {cross }}$ of sensitive K562 cells $(48.64 \mathrm{MHz})$ was chosen as working frequency of MDR detection unit [1]. This unit works based on electrohydrodynamic principles. Utilizing Newton's second law in the microchannel and assuming electroosmotic force and acceleration term as zero [9], the following inequality is obtained (Eq. 4), indicating that size and $R e\left(f_{C M}\right)$ of particles determine their trapping conditions under the same hydrodynamic settings.

$$
2 \pi \varepsilon_{\mathrm{m}} \mathrm{r}^{3} \operatorname{Re}\left(\mathrm{F}_{\mathrm{CM}}\right) \nabla \mathrm{E}_{\mathrm{rms}}^{2}>6 \pi \mu r v_{m}
$$

where, $v_{m}$ is the velocity of medium and. $\mu$ denotes the medium viscosity.

In this study, the average radius of drug sensitive and MDRK562 cells were $5.5 \mu \mathrm{m}$ while RBCs were $3 \mu \mathrm{m}$ in radius, determined by pixel measurement software (Pixel Ruler). Moreover, as it is shown in Figure $1, \operatorname{Re}\left(f_{C M}\right)$ of MDR-K562 cells were 1.52 times higher than RBCs. This implies that the DEP trapping force on MDR-K562 cells is 9.36 times higher than that of RBCs. If flow rate is optimized, the detection of MDR-K562 cells can be achieved in MDR detection unit with high selectivity.

Figure 3 and 4 show the fabrication flow and the fabricated device, respectively. Fabrication was started with dehydration of the 6" glass wafers which were cleaned with piranha and etched with BHF. Ti/Au was sputtered (BESTEC dual chamber sputter system) to form planar electrodes and a seed layer for $\mathrm{Cu}$ electroplating. Utilizing wet etching, planar electrodes and the seed layer were patterned. To prevent parasitic capacitances between $\mathrm{Au}$ layer (used to short nonconsecutive electrodes) and 3Delectrodes, a parylene layer $(\sim 0.5 \mu \mathrm{m})$ was coated (Parylene deposition system, SCS 2010 Labcoter $^{T M}$ 2).

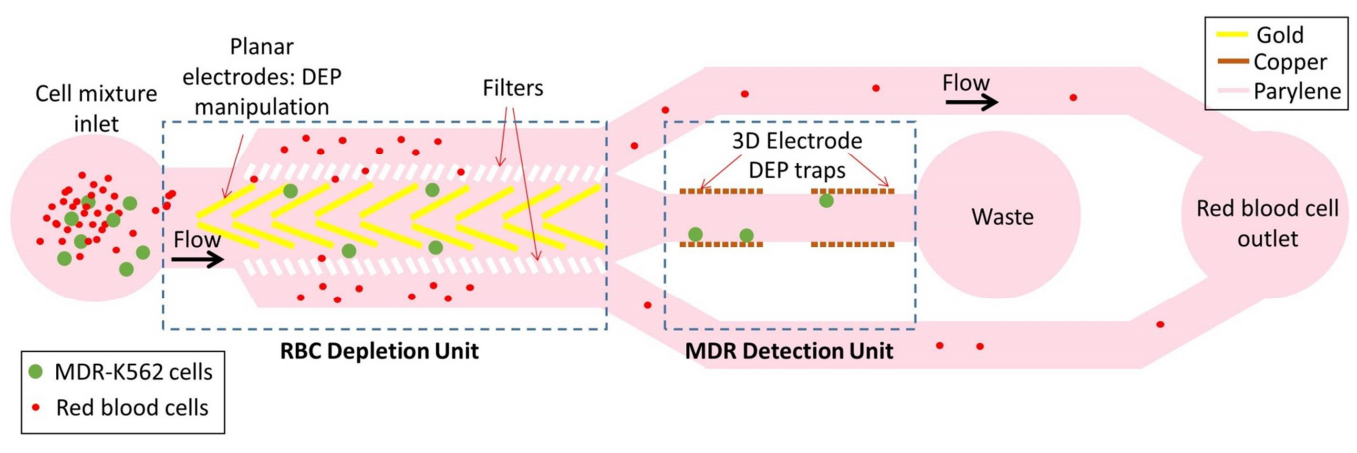

Figure 2: Device schematic. The system consists of 2 consecutive DEP units for the depletion of RBCs and capturing of MDR-K562 cells. 
3D-electrode places were patterned with parylene RIE (STS Multiplex RIE system). Cu-electroplating was carried out to obtain $20 \mu \mathrm{m}$ thick 3D-electrodes. Another parylene layer $(\sim 0.5 \mu \mathrm{m})$ was coated for insulation purposes. Next, the channel and parylene obstacles were formed with lithography and parylene coating $(\sim 20$ $\mu \mathrm{m})$. Finally, sacrificial photoresist was released with acetone.

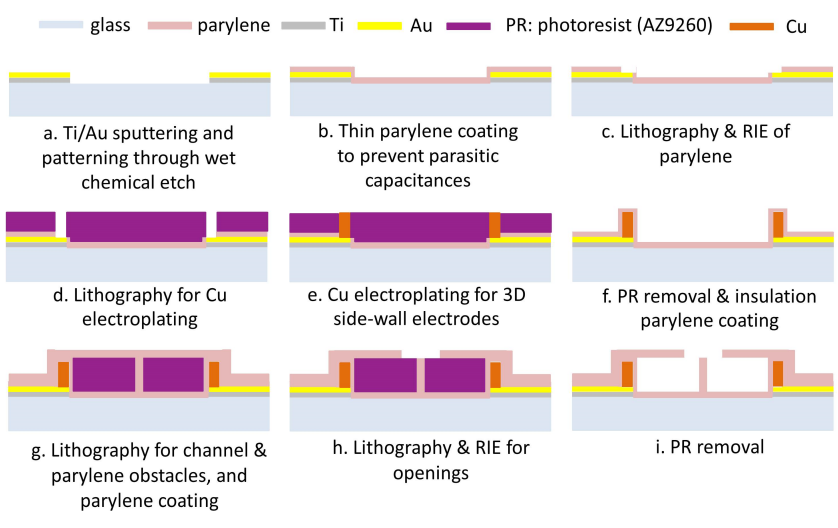

Figure 3: Fabrication flow.

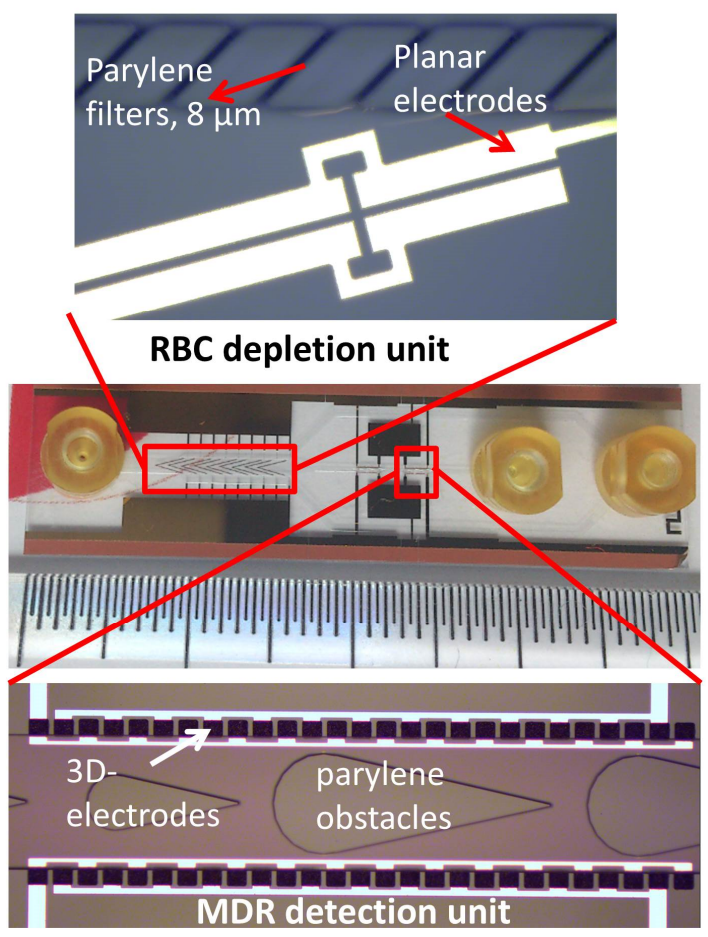

Figure 4: Fabricated device.

\section{EXPERIMENTAL}

\section{Materials}

The Philadelphia chromosome positive K562 cell lines were obtained from German Collection of Microorganisms and Cell Cultures, Germany, and cultured in RPMI 1640 medium (Invitrogen) with fetal bovine serum and penicillin-streptomycin. To develop resistance, doxorubicin (Saba) were added to different culture medium with gradual increment in their concentration until they become resistant to $1000 \mathrm{nM}$ doxorubicin [10]. Whole blood (3 $\mathrm{mL}$ ) was sampled from a healthy adult volunteer (woman) through venipuncture, utilizing EDTA treated tube to prevent coagulation. This sample was centrifuged at $900 \mathrm{rpm}$ for $15 \mathrm{~min}$. RBCs were harvested from the pellet.

\section{Test Setup and Procedure}

Tests were conducted under a fluorescent microscope (Olympus, SZX12). Observations were carried out under 20X magnification. A high-speed camera (Evolve 128) was utilized to observe the cell movement inside microchannel, under continuous flow and low fluorescence intensity. Flow rate was adjusted with a computer controlled microsyringe pump (Lab Smith). Real-time processing of screenshots was carried out with the computer software, Winfluor. DEP force was generated by energizing the electrode arrays with a signal generator (Agilent, 81150A). Two of its four outputs were utilized with $180^{\circ}$ phase difference. Figure 5 shows test setup.

Cell preparation and test procedure are as follows. MDRK562 cells and RBCs were centrifuged at $1000 \mathrm{rpm}$ for $5 \mathrm{~min}$ and $900 \mathrm{rpm}$ for $15 \mathrm{~min}$, respectively. The pellets were washed twice and suspended in an isotonic medium $(8.5 \%(\mathrm{w} / \mathrm{v})$ sucrose and 0.3 $\%(\mathrm{w} / \mathrm{v})$ dextrose) with a conductivity of $2.5 \mathrm{mS} / \mathrm{m}$. These medium properties were adapted from the study of Labeed et al. [4]. For monitoring purposes, RBCs were stained with cell tracker red (Invitrogen), a fluorescent dye with red color, and observed under red filter (31002 TRITC, Chroma). MDR-K562 cells were stained with fluorescein diacetate (Invitrogen), a fluorescent dye with green color, and observed under GFP filter. After 25 minutes incubation, cells were washed with the suspension medium. Finally, they were mixed and working cell mixture was obtained. The ratio of MDR-K562 cells and RBCs were adjusted as 2, 0.3, 0.1, 0.05, keeping MDR-K562 cell number constant.

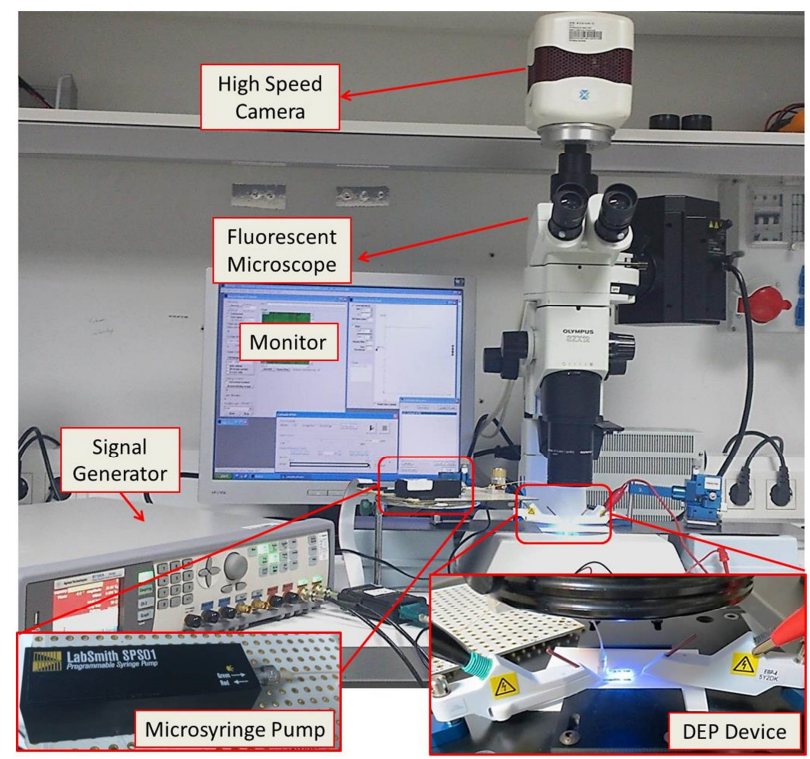

Figure 5: Test setup.

\section{RESULTS AND DISCUSSION}

Detection tests were performed with $20 \mathrm{~V}_{\mathrm{pp}}$ at a flow rate of $10 \mu \mathrm{l} / \mathrm{min}$. Figure 6 shows the screenshots of trapping tests of MDR-K562 cells for different cell ratios (video presentation is available). Table 1 presents the ratio of trapped MDR-K562 cells to RBCs. This ratio is infinite for the condition that MDR-K562 cells constitute less than $25 \%$ of overall population. This implies that MDR detection in K562 cells is achieved with $100 \%$ selectivity. 


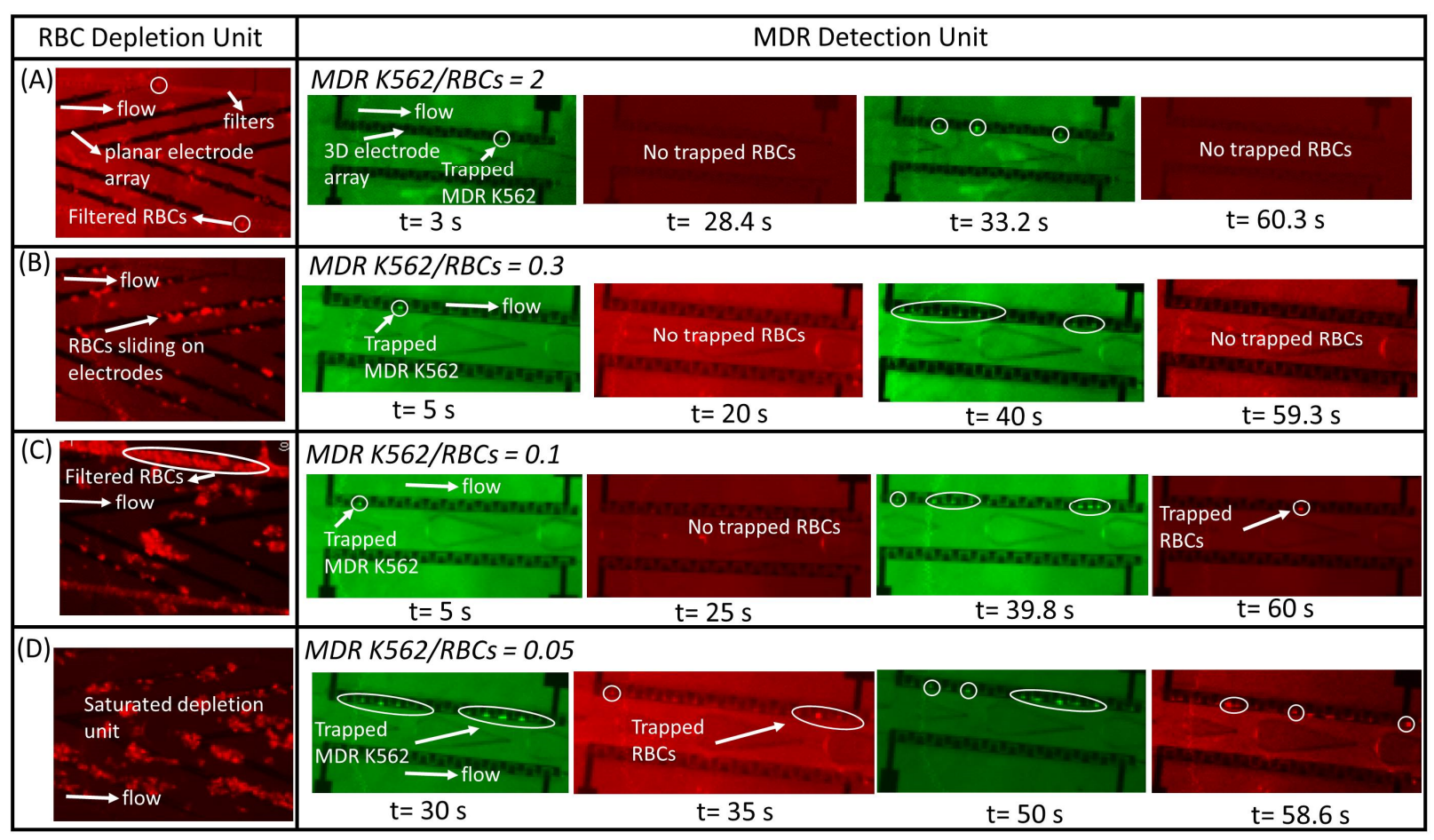

Figure 6: Pseudo-colored images taken during detection of MDR-K562 cells, green screens. Red screens show RBCs. Working solution was the mixture of MDR-K562 cells and RBCs with different concentrations ( $f=1 \mathrm{MHz}$ and $f=48.64 \mathrm{MHz}$ for depletion and detection units, respectively. Flow rate $=10 \mu \mathrm{l} / \mathrm{min}$, voltage $=20 \mathrm{~V}_{\mathrm{pp}}$ ).

On the other hand, increase in the number of RBCs was ensued with the trapping of them in MDR detection unit since $\mathrm{RBC}$ depletion unit was saturated and the harmony between electrical and hydrodynamic conditions was corrupted. This challenge can be overcome by increasing the length of RBC depletion unit.

Table 1: The ratio of trapped MDR-K562 cells to trapped RBCs for 4 cell mixtures, injected into channel with different ratios of MDR-K562 cells to RBCs.

\begin{tabular}{|c|c|c|c|c|}
\hline $\begin{array}{c}\text { The ratio of MDR-K562 to RBCs } \\
\text { in inlet }\end{array}$ & 2 & 0.3 & 0.1 & 0.05 \\
\hline $\begin{array}{c}\text { The ratio of trapped MDR-K562 } \\
\text { to RBCs }\end{array}$ & $\infty$ & $\infty$ & 4 & 1.75 \\
\hline
\end{tabular}

\section{ACKNOWLEDGEMENTS}

The authors acknowledge The Scientific and Technological Research Council of Turkey (TUBITAK) for the scholarship support for Mrs. Yağmur Demircan Yalçın and for the financial support through project 111E194.

*First and second authors have equal contributions on this study.

\section{REFERENCES}

[1] Y. Demircan, A. Koyuncuoğlu, M. Erdem, E. Özgür, U. Gündüz, and H. Külah, "Label-free detection of multidrug resistance in K562 cells through isolated 3D-electrode dielectrophoresis," Electrophoresis, 36, 1149-1157, (2015).

[2] M. Lehnert, "Clinical Multidrug Resistance in Cancer: A Multifactorial Problem," 32A, 912-920, (1996).

[3] J. Zhou (ed.), Multi-Drug Resistance in Cancer, Humana Press, New York, USA 2010.
[4] F. H. Labeed, H. M. Coley, H. Thomas, and M. P. Hughes, "Assessment of multidrug resistance reversal using dielectrophoresis and flow cytometry," Biophysical Journal, 85, 2028-2034, (2003).

[5] H.M. Coley, F. H. Labeed, H. Thomas, and M. P. Hughes, "Biophysical characterization of MDR breast cancer cell lines reveals the cytoplasm is critical in determining drug sensitivity," Biochimica et Biophysics Acta, 1770, 601-608, (2007).

[6] Y. Demircan, A. Koyuncuoğlu, M. Erdem, E. Özgür, U. Gündüz, and H. Külah, "Detection of imatinib resistance in K562 leukemia cells by 3D-electrode contactless dielectrophoresis," Technical Digest of the 2013 IEEE Transducers, 6/16-20/2013, Barcelona (2013), pp. 2086 2089.

[7] Y. Demircan, M. Erdem, E. Özgür, U. Gündüz, and H. Külah, "Determination of multidrug resistance level in K562 leukemia cells by 3D-electrode contactless dielectrophoresis," Technical Digest of the 2014 IEEE MEMS, 1/26-30/2014, San Francisco (2014), pp. 837 - 840.

[8] Y. Assef, S. M. Cavarra, A. E. Damiano, C. Ibarra, and B. Kotsias, "Ionic currents in multidrug resistant K562 human leukemic cells," Leukemia Research, 29, 1039-1047, (2005).

[9] B. Cetin and D. Li, "Dielectrophoresis in microfluidics technology," Electrophoresis, 32, 2410-27, (2011).

[10] Y. Baran, A. Ural, and U. Gündüz, U., "Mechanisms of cellular resistance to imatinib in human chronic myeloid leukemia cells," Hematology, 12, 497-503, (2007).

\section{CONTACT}

*Y.Demircan Yalçın, tel: +90-312-219-8312;

yagmur.demircan@mikrobiyo.com.tr. 